DIGITALCOMMONS @WAYNESTATE-
Clinical Research in Practice: The Journal of Team Hippocrates

\title{
Lifestyle intervention is superior to metformin in prevention of microvascular complications of diabetes in women, but socioeconomic barriers may lead to use of combination
}

\author{
Katanya C. Alaga \\ Wayne State University School of Medicine, Detroit, gh0766@wayne.edu \\ Jesse Haddad \\ Beaumont Hospital, Wayne, jesse.haddad@beaumont.org
}

Follow this and additional works at: https://digitalcommons.wayne.edu/crp

Part of the Dietetics and Clinical Nutrition Commons, Endocrinology, Diabetes, and Metabolism Commons, and the Family Medicine Commons

\section{Recommended Citation}

ALAGA KC, HADDAD J. Lifestyle intervention is superior to metformin in prevention of microvascular complications of diabetes in women, but socioeconomic barriers may lead to use of combination. Clin. Res. Prac. Oct 13 2021;7(2):eP2626. https://doi.org/10.22237/crp/1625098080

This Clinical Decision Report is brought to you for free and open access by the Open Access Journals at DigitalCommons@WayneState. It has been accepted for inclusion in Clinical Research in Practice: The Journal of Team Hippocrates by an authorized editor of DigitalCommons@WayneState. 


\section{Lifestyle intervention is superior to metformin in prevention of microvascular complications of diabetes in women, but socioeconomic barriers may lead to use of combination}

KATANYA C. ALAGA, MSc, Wayne State University School of Medicine, gh0766@wayne.edu

JESSE HADDAD, MD, Beaumont Hospital Wayne Family Medicine Residency Program, jesse.haddad@beaumont.org

\section{ABSTRACT A clinical decision report using:}

Diabetes Prevention Program Research Group. Long-term effects of lifestyle intervention or metformin on diabetes development and microvascular complications over 15-year follow-up: The Diabetes Prevention Program Outcomes Study. Lancet Diabetes Endocrinol. 2015;3(11):866-875. https://doi.org/10.1016/S2213-8587(15)00291-0

for a patient with pre-diabetes who is concerned about the prevention of microvascular complications through lifestyle intervention while managing her busy schedule as a traveling house cleaner.

Keywords: $\quad$ diabetes, lifestyle, microvascular complications, neuropathy, food-insecurity

\section{Clinical-Social Context}

Susan Berry (pseudonym) is a 57-year-old Caucasian female that presents to the family medicine clinic for follow up on her prediabetes. Ms. Berry was diagnosed with prediabetes three months prior with a A1c of 6.3 and has since attempted to manage her condition with diet and exercise. She has an additional medical history of hypertension, hyperlipidemia, and obesity (BMI $40.42 \mathrm{~kg} / \mathrm{m} 2$ ) that she manages with losartan, hydrochlorothiazide, and atorvastatin. She has no neuropathy, nephropathy, or retinopathy thus far. Ms. Berry is highly motivated to prevent the further development of diabetes and expressed her concern of watching her sister struggle with "constant pain and tingling in her feet" and "squeezing in" podiatry appointments in her busy schedule. She is also aware that diabetes can impair her vision and her kidneys and would also like to avoid these complications. She cites socioeconomic barriers including financial difficulty, few social supports, and time constraints to purchasing and preparing healthy meals with her employment as a traveling house cleaner. She currently has difficulty exercising and eats mostly fast food or frozen dinners because of her busy schedule. She is motivated to change her lifestyle and would like to avoid medications as much as possible. Her primary care team wondered about the comparative efficacy in lifestyle intervention or metformin to help Ms. Berry prevent the long-term diabetic complications that concern her while taking into consideration the socioeconomic barriers that she faces in this endeavor.

KATANYA C. ALAGA, MSc, is a student at the Wayne State University School of Medicine. JESSE HADDAD, MD, is in the Family Medicine Residency Program at Beaumont Hospital Waynei. 
ALAGA KC, HADDAD J. Lifestyle intervention is superior to metformin in prevention of microvascular complications of diabetes in women, but socioeconomic barriers may lead to use of combination. Clin. Res. Prac. Oct 13 2021;7(2):eP2626. https://doi.org/10.22237/crp/1625098080
Clinical Research in Practice The Journal of Team Hippocrates

VOL 7 ISS 2 / eP2626 / OCTOBER 13, 2021 https://doi.org/10.22237/crp/1625098080

\section{Clinical Question}

Does lifestyle intervention have a greater benefit than metformin in reducing long-term diabetic complications in patients with socioeconomic barriers to healthy living?

\section{Research Article}

Diabetes Prevention Program Research Group. Long-term effects of lifestyle intervention or metformin on diabetes development and microvascular complications over 15-year follow-up: The Diabetes Prevention Program Outcomes Study. Lancet Diabetes Endocrinol. 2015;3(11):866-875. https://doi.org/10.1016/S2213-8587(15)00291-0 ${ }^{1}$

\section{Description of Related Literature}

Utilizing PubMed Advanced Search Builder, title and abstract terms that included "metformin" AND "lifestyle" AND "diabetes" AND "adult" AND "complications" yielded 157 citations with the "best match filter". Subsequently, the randomized controlled trial filter was applied yielding 44 total citations since November 2020. The clinical queries and similar articles options on PubMed were utilized to ensure all relevant articles were reviewed. The abstracts of all 44 of these citations were preliminarily reviewed, and 33 were omitted because they were not relevant to our clinical question or did not involve populations that were related to our patient. For example, some were specific to pregnant women, HIV patients, obese adolescents etc. Of the remaining 11 articles reviewed, 6

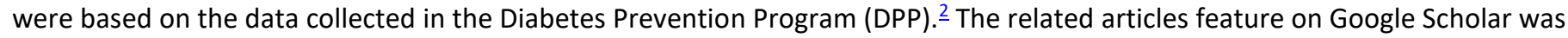
then used to source additional literature. The DPP, DPPOS, and ePREDICE trials were first to appear. Similar to Pubmed, other articles were reviews regarding the DPPOS trial or did not examine an applicable population of interest.

The DPP was a large, randomized control trial involving 3234 adults at high risk for development of type 2 diabetes. $\frac{2}{}$ The study had an average follow up of 2.8 years with a focus on diabetes incidence. The group was able to postulate that lifestyle intervention may possibly delay or prevent the development of diabetes-related complications. The articles examined in this query further examined the DPP to examine autonomic nervous system function $\underline{3}$, risk of progression to diabetes $\frac{4-6}{}$, the development of urinary incontinence ${ }^{-}$, and cardiovascular risk. ${ }^{-}$However, the duration of follow-up in the DPP was too short to evaluate these relationships in a clinically relevant way.

The DPP Outcomes Study (DPPOS) was a 15-year follow-up to the DPP that focused on microvascular complications related to diabetes. -1 Two articles selected for evaluation in our narrowed search examined the DPPOS for diabetes incidence- ${ }^{9}$ and depression. $\frac{10}{}$ The ePREDICE trial also published its protocol and recruitment for the early prevention of microvascular complications in hyperglycemic patients but have yet to finalize its results. $\underline{11}$ Based on these criteria, the DPPOS 1 is the most current study relevant to the concerns of our patient and is evaluating the most efficacious treatment plan to optimize her long-term prognosis. It is also the only randomized control trial examining the comparison between lifestyle intervention and metformin on the development of long-term microvascular complications which include quality of life-related outcomes such as neuropathy. As such, this study was examined to answer our clinical question and how its result could be applied in the clinical management of Ms. Berry.

Per the strength of recommendation taxonomy (SORT) $\frac{12}{2}$, this is a Grade of Recommendation Strength B based on the lack of multiple randomized control trials examining metformin compared to lifestyle for the prevention of microvascular diabetes related complications.

\section{Critical Appraisal}

The DPPOS 1 was a cohort study in which patients enrolled in the DPP² were evaluated in their respective treatment arms for 15 years of follow-up. The DPP was a randomized control trial that compared intensive lifestyle intervention with placebo or masked metformin in an adult cohort that was determined to be at high risk for the development of diabetes. At the end of the DPP, all participants were offered lifestyle training given to the lifestyle intervention group. $\underline{2}$ The DPPOS was very successful in maintaining a highly engaged cohort with $88 \%$ (2776) of the surviving DPP cohort being included. $\stackrel{1}{ }$ Per the SORT criteria, this is Level 2 evidence. $\underline{12}$ 
ALAGA KC, HADDAD J. Lifestyle intervention is superior to metformin in prevention of microvascular complications of diabetes in women, but socioeconomic barriers may lead to use of combination. Clin. Res. Prac. Oct 13 2021;7(2):eP2626. https://doi.org/10.22237/crp/1625098080
Clinical Research in Practice The Journal of Team Hippocrates

VOL 7 ISS 2 / eP2626 / OCTOBER 13, 2021 https://doi.org/10.22237/crp/1625098080

The DPPOS 1 observed there was no significant difference in the aggregate of microvascular outcomes in a patient who developed diabetes across all treatment arms. The number of cases of diabetes and cumulative incidence by year 15 were significantly lower by 27 and $18 \%$ with lifestyle intervention or with metformin, respectively, compared with the placebo group. The incidence of diabetes in the lifestyle, metformin, or placebo groups were 480 (55\%), 499 (56\%), and 560 (62\%), respectively. These results suggest that similar lifestyle intervention protocols may be beneficial in the potential reduction of long-term complications and could be beneficial to our patient in question. However, it should be noted that the outcome for women is a sub-group analysis and may not be a true difference.

The lifestyle intervention protocol instituted as part of the DPP included a goal to maintain a weight reduction of at least 7 percent of initial body weight through a healthy diet, and engagement in physical activity of moderate intensity for at least 150 minutes per

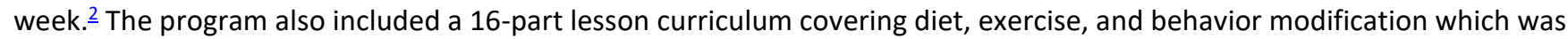
administered by personal case-managers one-on-one with patients during the first 24 weeks of enrollment. - The intensity and time commitment of this intervention brings into question the practicality of introducing a similar lifestyle intervention in reality. The intervention is particularly problematic when considering populations that resemble our patient of discussion who do not have the finances or time required to complete and maintain a similar program successfully. If this program were to be adapted to a more realistic approach it is unlikely that diet and exercise alone would be superior to medication. Hence the need in clinical practice for a combination of metformin as well as diet and exercise to combat microvascular complications.

Subsequent monthly individual and group sessions were offered to reinforce the behavioral change. During the DPP Outcomes Study all groups (metformin, lifestyle intervention, and placebo) were offered the lifestyle intervention program quarterly during a oneyear bridge period..$^{-}$This methodology resulted in a therapeutic cross-over between treatment arms. Further, the lifestyle intervention during the DPP Outcomes Study $\underline{1}$ was less intensive and resultant weight regain was observed in the lifestyle intervention group. The use of metformin by participants in the lifestyle and placebo groups may have also limited the magnitude of differences in microvascular outcomes among the three treatment arms. Once hyperglycemia worsened to FBG $\geq 7.78 \mathrm{mmol} / \mathrm{L}$ study drugs were discontinued, and diabetes management was transferred to a patient's health care provider. This introduced a biased selection of participants who have a certain degree of glycemic control, potentially again reducing the incidence of microvascular complications on its own.

The DPP Outcomes Study $\underline{1}$ demonstrated the long-term effect of lifestyle intervention and metformin to reduce the incidence of diabetes. However, after 15 years of enrollment the majority of individuals in each treatment arm had developed diabetes-related complications. Therefore, more effective interventions are necessary.

\section{Clinical Application}

The study demonstrates that while lifestyle intervention can significantly reduce the incidence of diabetes, its effectiveness in preventing long-term microvascular complications is less convincing. However, the study's findings of reduction in these complications in women show promise for patients such as ours. Ms. Berry was amenable to making lifestyle changes, but her socioeconomic difficulties and the lack of rigorous guided program support make this challenging. Unfortunately, while the patient is motivated, she is unable to commit to 150 minutes of exercise per week like the proposed program entails and is instead going to attempt 90 minutes a week on top of her busy schedule. The patient's concern about the possible increase in doctor's appointments if she developed additional symptoms of uncontrolled diabetes should be considered. Thus, the relative safety of metformin as well as our patient's goals and lifestyle played into the ultimate decision to use both interventions

The effectiveness of implementing a radical lifestyle intervention as described in the DPP Outcomes Study is questionable. However, the article did address our clinical question and its application can now be further examined under the scrutiny of food insecurity in at-risk persons and the recommendations to be made in the clinical setting for diabetes prevention. 
ALAGA KC, HADDAD J. Lifestyle intervention is superior to metformin in prevention of microvascular complications of diabetes in women, but socioeconomic barriers may lead to use of combination. Clin. Res. Prac. Oct 13 2021;7(2):eP2626. https://doi.org/10.22237/crp/1625098080
Clinical Research in Practice The Journal of Team Hippocrates

VOL 7 ISS 2 / eP2626 / OCTOBER 13, 2021 https://doi.org/10.22237/crp/1625098080

\section{New Knowledge Related to Clinical Decision Science}

Although outcomes in clinical research dichotomized metformin vs. lifestyle change, in practice, patients often prefer to apply both interventions given the socioeconomic barriers to intensive lifestyle changes alone. As such, in this risk-benefit discussion, particular attention was paid to the socioeconomic barriers Ms. Berry faces that could make the necessary lifestyle modifications very difficult if attempted alone. Research articles did not clearly show the outcome of using both, thus in the practical clinical setting we adapted the independent variable to a real-life application and strongly encouraged the patient to make lifestyle changes to the best of her ability in addition to using metformin. Further research is required to better understand how food insecurity and socioeconomic status preclude the beneficial effects observed through lifestyle intervention in the prevention of microvascular consequences of diabetes. Narrowing approaches to care of diabetic patients based on specific research alone does a disservice to patients' long-term quality of life and can hinder the achievement of their goals.

\section{Conflict Of Interest Statement}

The authors declare no conflict of interest.

\section{References}

1. Diabetes Prevention Program Research Group. Long-term effects of lifestyle intervention or metformin on diabetes development and microvascular complications over 15-year follow-up: The Diabetes Prevention Program Outcomes Study. Lancet Diabetes Endocrinol. 2015;3(11):866-875. https://doi.org/10.1016/S2213-8587(15)00291-0

2. Diabetes Prevention Program Research Group. Reduction of the incidence of type 2 diabetes with lifestyle intervention or metformin. N Engl J Med. 2002;346(6):393-403. https://doi.org/10.1056/nejmoa012512

3. Carnethon MR, Prineas RJ, Temprosa M, Zhang ZM, Uwaifo G, Molitch ME. The association among autonomic nervous system function, incident diabetes, and intervention arm in the diabetes prevention program. Diabetes Care. 2006;29(4):914-919. https://doi.org/10.2337/diacare.29.04.06.dc05-1729

4. Herman WH, Pan Q, Edelstein SL, et al. Impact of lifestyle and metformin interventions on the risk of progression to diabetes and regression to normal glucose regulation in overweight or obese people with impaired glucose regulation. Diabetes Care. 2017;40(12):1668-1677. https://doi.org/10.2337/dc17-1116

5. DeBoer MD, Filipp SL, Gurka MJ. Use of a metabolic syndrome severity z score to track risk during treatment of prediabetes: An analysis of the diabetes prevention program. Diabetes Care. 2018;41(11):2421-2430. https://doi.org/10.2337/dc18-1079

6. Florez H, Temprosa MG, Orchard TJ, et al. Metabolic syndrome components and their response to lifestyle and metformin interventions are associated with differences in diabetes risk in persons with impaired glucose tolerance. Diabetes, Obes Metab. 2014;16(4):326-333. https://doi.org/10.1111/dom.12220

7. Brown JS, Wing R, Barrett-Connor $E$, et al. Lifestyle intervention is associated with lower prevalence of urinary incontinence: The diabetes prevention program. Diabetes Care. 2006;29(2):385-390. https://doi.org/10.2337/diacare.29.02.06.dc05-1781

8. Goldberg RB, Temprosa M, Haffner S, et al. Effect of progression from impaired glucose tolerance to diabetes on cardiovascular risk factors and its amelioration by lifestyle and metformin intervention. Diabetes Care. 2009;32(4):726-732. https://doi.org/10.2337/dc08-0494

9. Diabetes Prevention Program Research Group. 10-year follow-up of diabetes incidence and weight loss in the Diabetes Prevention Program Outcomes Study. Lancet. 2009;374(9702):1677-1686. https://doi.org/10.1016/S0140-6736(09)61457-4

10. Marrero DG, Ma Y, De Groot M, et al. Depressive symptoms, antidepressant medication use, and new onset of diabetes in participants of the diabetes prevention program and the diabetes prevention program outcomes study. Psychosom Med. 2015;77(3):303-310. https://doi.org/10.1097/PSY.0000000000000156

11. Gabriel R, Abdelkader NB, Acosta T, et al. Early prevention of diabetes microvascular complications in people with hyperglycaemia in Europe. ePREDICE randomized trial. Study protocol, recruitment and selected baseline data. PLoS One. 2020;15(4):e0231196. https://doi.org/10.1371/journal.pone.0231196

12. Ebell MH, Siwek J, Weiss BD, et al. Strength of Recommendation Taxonomy (SORT): A Patient-Centered Approach to Grading Evidence in the Medical Literature. The Journal of the American Board of Family Practice. 2004;17(1):59-67.

https://doi.org/10.3122/jabfm.17.1.59 